Proceedings of the International School and Conference on Optics and Optical Materials, ISCOM07, Belgrade, Serbia, September 3-7, 2007

\title{
Optical and Electron Spectrometry of Molecules of Biological Interest
}

\author{
B.P. Marinković ${ }^{a, b, *}$, A.R. Milosavluević ${ }^{a}$, \\ J.B. Maljkovića ${ }^{a}$, D. Ševića ${ }^{a, b}$, B.A. Petruševski ${ }^{b}$, \\ D. Pavlovića ${ }^{a}$, D.M. Filipović ${ }^{a, c}$, M. Terzić ${ }^{d}$ \\ AND V. PEJČEV ${ }^{a, e}$ \\ ${ }^{a}$ Institute of Physics, Pregrevica 118, 11080 Belgrade, Serbia \\ ${ }^{b}$ Electrical Engineering College, Belgrade, Serbia \\ ${ }^{c}$ Faculty of Physics, University of Belgrade, Serbia \\ ${ }^{d}$ Faculty of Sciencies, University of Novi Sad, Serbia \\ ${ }^{e}$ Faculty of Sciences, University of Kragujevac, Serbia
}

Optical absorption and emission spectroscopy together with low energy electron interaction (elastic scattering, excitation, ionization, resonances) with biologically relevant molecules (nitrogen, oxygen, water, alcohols, tetrahydrofuran, tetrahydrofurfuril alcohol, 3-hydroxytetrahydrofuran, pyrimidine, glycine, alanine) are studied in order to understand radiation damage and to investigate the presence of pollutants in the atmosphere. Versatile high resolution electron spectrometers are used in the present study of electron-molecule interactions. Energy loss spectra were recorded for these molecules in order to identify electronic transitions from ground state to both allowed and optically forbidden states. Optical emission spectra have been recorded from gas discharge processes by low resolution optical spectrometer (Ocean Optics 2000). Also, electronic spectra were compared with high resolution synchrotron photoabsorption spectra where these spectra had been available. Experimental methods of absorption-based laser spectroscopy were reviewed being of the most widely used analytical tools for detection of a specific molecule and quantitative measurements, based on the Beer-Lambert absorption law.

PACS numbers: 34.80.Gs, 34.80.Bm, 52.25.Os

${ }^{*}$ corresponding author; e-mail: bratislav.marinkovic@phy.bg.ac.yu 


\section{Introduction}

Optical absorption and emission spectroscopy together with electron energy loss spectroscopy (EELS) of biologically relevant molecules represent the most important tools in investigating the elementary processes between quantum particles at molecular level. Optical spectroscopy has an advantage over EELS in higher achievable energy resolution that could reveal both electronic and ro-vibrational transitions. However, it is limited to allowed dipole transitions. But in EELS, contrary to optical spectroscopy, by varying the incident electron energy and the scattering angle at which spectra are obtained, both allowed and forbidden transitions may be observed. Another advantage of electron spectroscopy lies in the accessibility of broader range of excitation energies. By different design and tuning the electron lenses of electron spectrometer, it is easy to reach any incident energy range: low (0.5 to $10 \mathrm{eV})$, intermediate $(10-100 \mathrm{eV})$ and high (over $100 \mathrm{eV})$. In optical spectroscopy, to go beyond the visible excitation range (roughly from 1.5 to $4 \mathrm{eV}$ ) one needs sophisticated instruments like synchrotron radiation sources in order to achieve higher excited states.

Motivation for the present study of molecules of biological interest exists in many areas of human activities. Besides gaining the fundamental knowledge of interactions between projectile particle (photon/electron) and molecules, better understanding of fundamental processes is needed in areas of investigation like environmental studies [1], radiation damage of DNA and other cellular components [2], optical plasma diagnostics [3], etc. Also, there are recent results that indicate that both UV and electron radiation of ice mixtures of organic molecules (a gas mixture deposited on a cold surface at about $10 \mathrm{~K}$ ) can lead to the formation of the simplest amino acids such as glycine and alanine [4].

Another aim of the present studies of organic molecules is to investigate the presence of pollutants. Different laser spectroscopy methods are available for these purposes. Absorption-based laser spectroscopy is one of the most widely used analytical tools for detection of a specific molecule and one of the most important techniques for gas sensors in environmental [5]. Direct laser absorption spectroscopy based on the Beer-Lambert absorption law is often used for quantitative measurements.

\section{Electron spectrometers and experimental procedure}

The first spectrometer ESMA, described in detail elsewhere [6], consists of the titanium oven heated by coaxial heater and hemispherical-energy selectors both in monochromator and analyzer as well as corresponding cylindrical lenses for accelerating, decelerating, and focusing the electron beam. The scattered electrons were energy analyzed by the selector, and were detected by a single-channel electron multiplier. The energy analyzer can be rotated around the atomic beam axis from $-30^{\circ}$ to $+150^{\circ}$. Differential pumping of both the electron monochromator and the electron energy analyzer was used. The background pressure was 
about $2 \times 10^{-7} \mathrm{mbar}$. The true zero scattering angle was determined from the symmetry of intense resonant inelastic feature's signal in the negative and positive angles. Energy loss spectra were recorded at several fixed energies from $10 \mathrm{eV}$ to $100 \mathrm{eV}$. The overall resolution was from $50 \mathrm{meV}$ to $100 \mathrm{meV}$. The effusive molecular beam was produced from amino acids in form of a powder. The temperature of the oven was monitored by two thermo-pairs, one placed at the bottom of the oven, the other at the top. The difference of temperature was constantly maintained to about $50 \mathrm{~K}$.

The second spectrometer UGRA, described in detail elsewhere [7], uses an electron gun that gives $100 \mathrm{nA}$ electron current and that could be rotated around a molecular beam axis from $-30^{\circ}$ to $+130^{\circ}$. The effusive molecular beam has been produced by a stainless steel needle. The base pressure was about $3 \times 10^{-7}$ mbar (turbomolecular pump) and the operating pressure was about $6 \times 10^{-6}$ mbar. Anhydrous liquid samples with a stated purity better of $+99.8 \%$ were used after several cycles of freeze-thaw under vacuum. The effective path length correction was checked against benchmark differential cross-sections for $\mathrm{Ar}$ and $\mathrm{Kr}$ and was found to be negligible in the covered angular range $\left(30^{\circ}\right.$ to $\left.110^{\circ}\right)$. The uncertainty of the angular scale was found to be less than $0.5^{\circ}$.

\section{Electron energy loss spectra}

Energy loss spectra of several bio-molecules has been recorded in order to obtain the broader picture of the excitation processes, energy deposition channels in the high-energy particle passage through cellular media and recognize electron induced DNA (and its constituents) damage. One of the first studies of this kind had been performed on plasmid DNA at the University of Sherbrooke [8] and had resulted with the determination of the cross-sections for the single strand breaks at very low incident electron energies [9]. We have undertaken a series of measurements on both DNA analogue molecules and simple amino acids [10]. A new set of results are presented here.

\subsection{DNA deoxyribose analogue molecules (tetrahydrofuran, tetrahydrofurfuril alcohol and 3-hydroxytetrahydrofuran)}

Preliminary results of electron interactions with DNA deoxyribose analogue molecules (tetrahydrofuran (THF) and tetrahydrofurfuril alcohol (THFA)) have been presented recently in the Progress Report paper by Milosavljević [11]. Investigation of electron interaction with DNA components is important to better understand processes driven by low-energy secondary electrons, which are produced in large quantities on the track of a primary high-energy ionizing particle. Here we present the results for another analogue molecule 3-hydroxytetrahydrofuran, $\mathrm{C}_{4} \mathrm{H}_{8} \mathrm{O}_{2}$ (3hTHF). Energy loss spectrum of 3hTHF is presented in Fig. 1. Spectrum shows several broad features in the recorded energy-loss domain from 4 to $15 \mathrm{eV}$. The present EEL spectrum is in a very good agreement with the positions of the peaks obtained in the high resolution VUV photoabsorption spectrum [12]. 


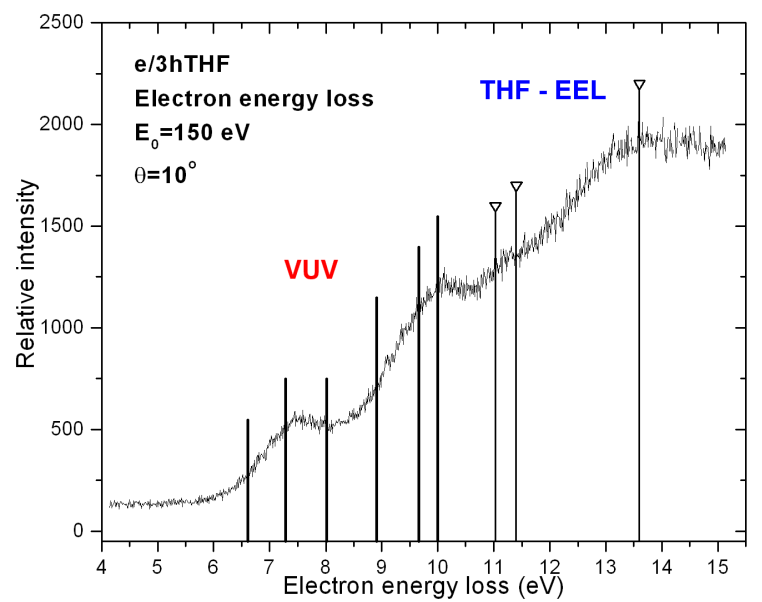

Fig. 1. Energy loss spectrum of $3 \mathrm{hTHF}$ at $150 \mathrm{eV}$ electron impact energy and $10^{\circ}$ scattering angle. Relative intensities had not been corrected for the energy transmission of the analyzer. Vertical bars represent the positions of the peaks obtained in the high resolution VUV photoabsorption spectrum of 3hTHF [12]. Down triangles represent the positions of the peaks obtained in the electron energy loss spectrum of THF molecule [11].

According to our knowledge, there are no previous EELS results for 3hTHF. Therefore, above $10 \mathrm{eV}$ we only indicate the positions that correspond to the higher excitation energies of THF molecule in EELS [11] (down triangles in Fig. 1).

\subsection{Amino acids}

The amino acids are the building blocks of peptides and proteins and they consist of two characteristic groups - amino group $\left(-\mathrm{NH}_{2}\right)$ and carboxyl group $(-\mathrm{COOH})$. The simplest amino acid is glycine $\left(\mathrm{H}_{2} \mathrm{~N}-\mathrm{CH}_{2}-\mathrm{COOH}\right)$ while alanine could be considered as a parent molecule for the other amino acids since the replacement of one or two $\mathrm{H}$ atoms in the methyl group with some radical leads to the structural formulae of other amino acids.

Here we present the results for alanine molecule energy loss spectrum (Fig. 2) recorded at $80 \mathrm{eV}$ electron impact energy and scattering angle of $2^{\circ}$. Spectrum shows several broad features, two of them identified at 6.29 and $7.28 \mathrm{eV}$ energy loss. Alanine came as a powder and the producing of the effusive beam is maintained through the process of sublimation. High resolution energy loss spectra of glycine and alanine has been recently presented by Abouaf [13].

\section{Absorption-based laser spectroscopy and optical emission spectroscopy}

Optoacoustic spectroscopy is based on the optoacoustic (OA) effect, in which acoustic waves result from the absorption of laser radiation by a selected target. 


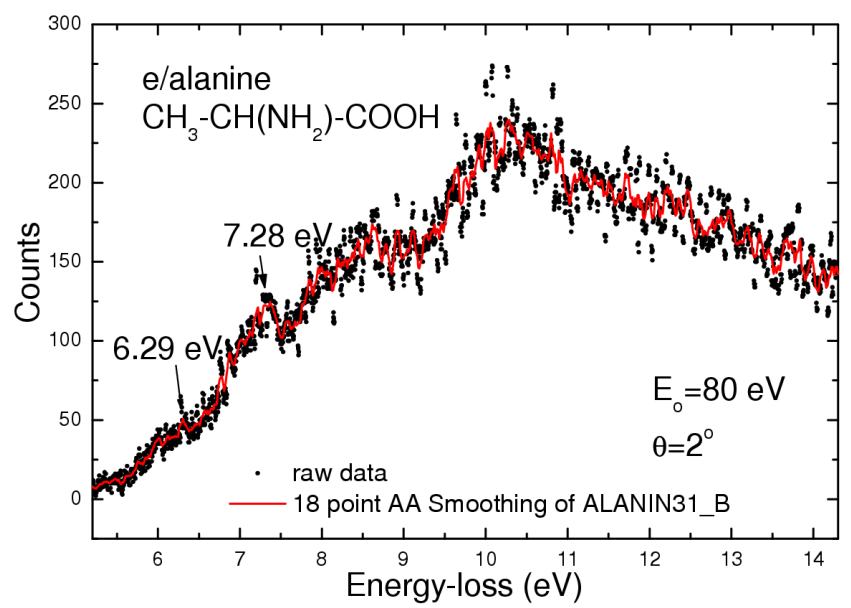

Fig. 2. Energy loss spectrum of alanine recorded at $80 \mathrm{eV}$ impact electron energy and $2^{\circ}$ scattering angle.Points are raw data and solid curve represents the 18 point smoothing curve of the original spectrum..

Light absorption results in a transient temperature effect, which then transforms into kinetic energy or pressure variations in the absorbing medium via nonradiative relaxation processes. OA technique had been successfully used to detect multiphoton processes in the presence of buffer gas mixtures [14]. Optical emission spectra have been recorded from gas discharge processes by low resolution optical spectrometer (Ocean Optics 2000), for nitrogen and several molecules [15].

\section{Acknowledgments}

The work was done within the project OI 141011 financed by the Ministry of Science, Republic of Serbia. We are pleased to recognize support of EU/ESF COST Action P9 on Radiation Damage and the European Science Foundation EIPAM Programme.

\section{References}

[1] N.J. Mason, A. Dawes, R. Mukerji, E.A. Drage, E. Vasekova, S.M. Webb, P. Limão-Vieira, J. Phys. B, At. Mol. Opt. Phys. 38, S893 (2005).

[2] N.J. Mason, M.A. Smialek, S.A. Moore, M. Folkard, S.V. Hoffmann, in: Proc. XXIII SPIG Int. Conf. and Symp. on Phys. of Ionized Gases, Kopaonik (Serbia), Eds. Lj. Hadžievski, B.P. Marinković, N.S. Simonović, American Institute of Physics, Conference Proceedings 876, Invited Lecture, Melville, New York2006, p. 3 .

[3] J.B. Boffard, C.C. Lin, C.A. DeJoseph Jr, J. Phys. D, Appl. Phys. 37, R143 (2004).

[4] G.M. Muños-Caro, U.J. Meierhenrich, W.A. Schutte, B. Barbier, A. Arcones Segovia, H. Rosenbauer, W.H.-P. Thiemann, A. Brack, J.M. Greenberg, Nature 416, 403 (2002). 
[5] M. Terzić, J. Možina, D. Horvat, Facta Universitatis, Series: Phys., Chem. Technol. 4, 71 (2006).

[6] S. Milisavljević, D. Šević, V. Pejčev, D.M. Filipović, B.P. Marinković, J. Phys. B, At. Mol. Opt. Phys. 37, 3571 (2004).

[7] A.R. Milosavljević, S. Madžunkov, D. Šević, I. Čadež, B.P. Marinković, J. Phys. B, At. Mol. Opt. Phys. 39, 609 (2006).

[8] B. Boudaiffa, P. Cloutier, D. Hunting, M.A. Huels, L. Sanche, Science 287, 1658 (2000).

[9] R. Panajotović, F. Martin, P. Cloutier, D. Hunting, L. Sanche, Radiat. Res. 165, 452 (2006).

[10] B.P. Marinković, D.M. Filipović, V. Pejčev, D. Šević, A.R. Milosavljević, D. Pavlović, S. Milisavljević, P. Kolarž, M. Pardjovska, in: Proc. XXIV ICPEAC Int. Conf. Photonic, El. and At. Collisions, Rosario (Argentina), Ed. P.D. Fainstein, World Scientific, Singapore 2006, p. 336.

[11] A.R. Milosavljević, in: Proc. XXIII SPIG Int. Conf. and Symp. on Phys. of Ionized Gases, Kopaonik (Serbia), Eds. Lj. Hadžievski, B.P. Marinković, N.S. Simonović, American Institute of Physics, Conference Proceedings 876, Progress Report, Melville, New York 2006, p. 80 and references therein.

[12] P. Limão-Vieira, M.-J. Hubin-Franskin, private communication.

[13] R. Abouaf, in: Proc. EIPAM07 Third Annual Meeting, European Science Foundation, Hveragerdi (Iceland) 2007.

[14] D.D. Markushev, M. Terzić, J. Jovanović-Kurepa, Rev. Sci. Instrum. 74, 303 (2003).

[15] D.M. Filipović, private communication. 